\title{
ON MIXED FINITE ELEMENT METHODS FOR THE REISSNER-MINDLIN PLATE MODEL
}

\author{
RICARDO DURÁN AND ELSA LIBERMAN
}

\begin{abstract}
In this paper we analyze the convergence of mixed finite element approximations to the solution of the Reissner-Mindlin plate problem. We show that several known elements fall into our analysis, thus providing a unified approach. We also introduce a low-order triangular element which is optimalorder convergent uniformly in the plate thickness.
\end{abstract}

\section{INTRODUCTION}

We consider the approximation by mixed finite elements of the solution to the Reissner-Mindlin equations, which describe the displacement of a plate with small to moderate thickness subject to a transverse load.

As is well known, standard finite element methods fail to give good approximations when the plate thickness is too small, owing to a locking phenomenon. Instead, mixed methods, based on the introduction of the shear strain as a new variable, have been proven successful both theoretically and experimentally [2, $4,5,6,7,9,10]$.

In this paper we analyze the convergence of mixed approximations for the plate problem in a general framework. We obtain a general convergence theorem, which can be applied to several elements, thereby providing a unified approach. For the Bathe-Dvorkin elements [6] our theorem provides an optimalorder error estimate under weaker regularity assumptions than those required in [4] (although still not optimal). Also, it can be applied to the higher-order elements introduced by Bathe and Brezzi [5], extending the estimates obtained by these authors in the limit case (thickness equal to 0 ).

Recently, Arnold and Falk [2] proposed and analyzed a low-order triangular element. Their analysis is based on an equivalence between the plate equations and an uncoupled system of two Poisson equations plus a Stokes-like system. This equivalence was first introduced by Brezzi and Fortin in [9] and was obtained by using a Helmholtz decomposition of the shear strain. Arnold and Falk proved optimal-order convergence uniformly in the plate thickness for their elements by introducing a discrete version of the Helmholtz decomposition. Our analysis provides a direct proof of the convergence of the Arnold-Falk elements without using the discrete Helmholtz decomposition.

Received February 22, 1990; revised October 24, 1990.

1991 Mathematics Subject Classification. Primary 65N30, 73K10, 73V05.

Key words and phrases. Reissner, Mindlin, mixed finite elements.

The first author's research was performed under a grant of Fundación Antorchas, República Argentina. The second author is a member of CIC, Provincia de Buenos Aires, Argentina. 
We also introduce a new low-order triangular element for which we prove optimal error estimates independently of the plate thickness.

\section{Statement of THE PROBlem AND NOtATIONS}

We use standard notation for the Sobolev spaces $H^{k}(\Omega)$ and $H_{0}^{k}(\Omega)$ with the norm

$$
\|u\|_{k}^{2}=\sum_{|\alpha| \leq k}\left\|D^{\alpha} u\right\|_{L^{2}(\Omega)}^{2},
$$

both for scalar and vector functions. Boldface type is used to denote vector quantities.

Let $\Omega \times\left(-\frac{t}{2}, \frac{t}{2}\right)$ be the region occupied by the undeformed plate, where $\Omega \subset \mathbb{R}^{2}$ is a simply connected polygon and $0<t<1$ is the plate thickness.

Let us denote by $w$ and $\boldsymbol{\beta}$ the transverse displacement of the midsection of the plate and the rotation of fibers normal to it, respectively. Then, for homogeneous Dirichlet boundary conditions (i.e., a clamped plate) the ReissnerMindlin model states that $\boldsymbol{\beta} \in \mathbf{H}_{0}^{1}(\Omega)$ and $w \in H_{0}^{1}(\Omega)$ satisfy

$$
t^{3} a(\boldsymbol{\beta}, \boldsymbol{\eta})+\lambda t(\boldsymbol{\nabla} w-\boldsymbol{\beta}, \boldsymbol{\nabla} v-\boldsymbol{\eta})=(g, v)
$$

for every $\boldsymbol{\eta} \in \mathbf{H}_{0}^{1}(\Omega)$ and $v \in H_{0}^{1}(\Omega)$, where (, ) denotes the scalar product in either $L^{2}(\Omega)$ or $\mathbf{L}^{2}(\Omega)$, and

$$
\begin{aligned}
a(\boldsymbol{\beta}, \boldsymbol{\eta})=\frac{E}{12\left(1-\nu^{2}\right)} \int_{\Omega}\left[\left(\frac{\partial \beta_{1}}{\partial x_{1}}+\right.\right. & \left.\nu \frac{\partial \beta_{2}}{\partial x_{2}}\right) \frac{\partial \eta_{1}}{\partial x_{1}}+\left(\nu \frac{\partial \beta_{1}}{\partial x_{1}}+\frac{\partial \beta_{2}}{\partial x_{2}}\right) \frac{\partial \eta_{2}}{\partial x_{2}} \\
& \left.+\frac{1-\nu}{2}\left(\frac{\partial \beta_{1}}{\partial x_{2}}+\frac{\partial \beta_{2}}{\partial x_{1}}\right)\left(\frac{\partial \eta_{1}}{\partial x_{2}}+\frac{\partial \eta_{2}}{\partial x_{1}}\right)\right],
\end{aligned}
$$

where $E$ is the Young modulus, $\nu$ the Poisson ratio, $\lambda=E k / 2(1+\nu)$ where $k$ is a constant, and $g$ represents the transverse load.

It is known that $a($,$) is coercive in \mathbf{H}_{0}^{1}(\Omega)$, and so it defines a scalar product in this space equivalent to the usual one.

In order to analyze the behavior of the approximations for small values of $t$, it is natural [4] to consider a load of the form $g=t^{3} f$. Then, if $\lambda=1$ for the sake of simplicity, problem (2.1) reduces to finding $\beta \in \mathbf{H}_{0}^{1}(\Omega)$ and $w \in H_{0}^{1}(\Omega)$ such that

$$
a(\boldsymbol{\beta}, \boldsymbol{\eta})+t^{-2}(\boldsymbol{\nabla} w-\boldsymbol{\beta}, \boldsymbol{\nabla} v-\boldsymbol{\eta})=(f, v)
$$

for every $\boldsymbol{\eta} \in \mathbf{H}_{0}^{1}(\Omega)$ and $v \in H_{0}^{1}(\Omega)$, or equivalently,

$$
a(\boldsymbol{\beta}, \boldsymbol{\eta})+(\boldsymbol{\gamma}, \boldsymbol{\nabla} v-\boldsymbol{\eta})=(f, v)
$$

and

$$
\boldsymbol{\gamma}=t^{-2}(\boldsymbol{\nabla} w-\boldsymbol{\beta})
$$

for every, $\boldsymbol{\eta} \in \mathbf{H}_{0}^{1}(\Omega)$ and $v \in H_{0}^{1}(\Omega)$.

In what follows we denote by $C$ a constant independent of $h$ and $t$ but not necessarily the same at each occurrence.

\section{MiXed FINITE ELEMENT APPROXIMATIONS AND ERROR ANALYSIS}

Let $\left\{\mathscr{T}_{h}\right\}_{0<h<1}$ be a regular family of triangulations of $\Omega$ [11], and let $\mathbf{H}_{h}, W_{h}$, and $\boldsymbol{\Gamma}_{h}$ be finite element spaces associated with $\mathscr{T}_{h}$ such that

$$
\mathbf{H}_{h} \subset \mathbf{H}_{0}^{1}(\Omega), \quad W_{h} \subset H_{0}^{1}(\Omega), \quad \text { and } \quad \Gamma_{h} \subset \mathbf{L}^{2}(\Omega) .
$$


We assume that

$$
\nabla W_{h} \subset \Gamma_{h} .
$$

A mixed approximation to the solution of problem (2.3) is obtained by relaxing the equation (2.3b) by means of a projection or interpolation over $\boldsymbol{\Gamma}_{h}$.

Assume that we have defined an operator $\Pi: \mathbf{V} \rightarrow \Gamma_{h}$, where $\mathbf{H}_{0}^{1}(\Omega) \subset \mathbf{V} \subset$ $\mathbf{L}^{2}(\Omega)$, such that

$$
\|\boldsymbol{\eta}-\boldsymbol{\Pi} \boldsymbol{\eta}\|_{0} \leq C h\|\boldsymbol{\eta}\|_{1}
$$

for every $\boldsymbol{\eta} \in \mathbf{H}^{\mathbf{1}}(\boldsymbol{\Omega}) \cap \mathbf{V}$.

Then, we define $\left(\boldsymbol{\beta}_{h}, w_{h}, \boldsymbol{\gamma}_{h}\right) \in \mathbf{H}_{h} \times W_{h} \times \boldsymbol{\Gamma}_{h}$ by

$$
a\left(\boldsymbol{\beta}_{h}, \boldsymbol{\eta}\right)+\left(\boldsymbol{\gamma}_{h}, \boldsymbol{\nabla} v-\boldsymbol{\Pi} \boldsymbol{\eta}\right)=(f, v)
$$

and

$$
\boldsymbol{\gamma}_{h}=t^{-2}\left(\boldsymbol{\nabla} w_{h}-\boldsymbol{\Pi} \boldsymbol{\beta}_{h}\right)
$$

for every $\boldsymbol{\eta} \in \mathbf{H}_{h}$ and $v \in W_{h}$.

The existence and uniqueness of the solution follows easily from the coerciveness of $a($,$) .$

Remark 3.1. The approximate solutions could be defined even if (3.1) is not satisfied. In that case we should replace $\nabla v$ and $\nabla w_{h}$ by $\Pi(\nabla v)$ and $\Pi\left(\nabla w_{h}\right)$, respectively, in (3.3) [10].

However, since in all of our applications (3.1) holds, we will not analyze the more general case.

From (2.3a) we obtain

$$
a(\boldsymbol{\beta}, \boldsymbol{\eta})+(\boldsymbol{\gamma}, \boldsymbol{\nabla} v-\boldsymbol{\Pi} \boldsymbol{\eta})=(f, v)-(\boldsymbol{\gamma}, \boldsymbol{\Pi} \boldsymbol{\eta}-\boldsymbol{\eta})
$$

for every $\boldsymbol{\eta} \in \mathbf{H}_{0}^{1}(\mathbf{\Omega})$ and $v \in H_{0}^{1}(\Omega)$ and, subtracting (3.3a) from this equation, we get the error equation

$$
a\left(\boldsymbol{\beta}-\boldsymbol{\beta}_{h}, \boldsymbol{\eta}\right)+\left(\boldsymbol{\gamma}-\boldsymbol{\gamma}_{h}, \boldsymbol{\nabla v}-\boldsymbol{\Pi} \boldsymbol{\eta}\right)=(\boldsymbol{\gamma}, \boldsymbol{\eta}-\boldsymbol{\Pi} \boldsymbol{\eta})
$$

for every $\boldsymbol{\eta} \in \mathbf{H}_{h}$ and $v \in W_{h}$.

Lemma 3.1. Let $\hat{\boldsymbol{\beta}} \in \mathbf{H}_{h}, \hat{w} \in W_{h}$, and $\hat{\boldsymbol{\gamma}}=t^{-2}(\boldsymbol{\nabla} \hat{w}-\boldsymbol{\Pi} \hat{\boldsymbol{\beta}}) \in \boldsymbol{\Gamma}_{h} ;$ then,

$$
\left\|\hat{\boldsymbol{\beta}}-\boldsymbol{\beta}_{h}\right\|_{1}+t\left\|\hat{\boldsymbol{\gamma}}-\boldsymbol{\gamma}_{h}\right\|_{0} \leq C\left\{\|\hat{\boldsymbol{\beta}}-\boldsymbol{\beta}\|_{1}+t\|\hat{\boldsymbol{\gamma}}-\boldsymbol{\gamma}\|_{0}+h\|\boldsymbol{\gamma}\|_{0}\right\} \text {. }
$$

Proof. From (3.4) we get

$$
\begin{aligned}
& a\left(\hat{\boldsymbol{\beta}}-\boldsymbol{\beta}_{h}, \boldsymbol{\eta}\right)+\left(\hat{\boldsymbol{\gamma}}-\boldsymbol{\gamma}_{h}, \nabla v-\Pi \boldsymbol{\eta}\right) \\
& \quad=a(\hat{\boldsymbol{\beta}}-\boldsymbol{\beta}, \boldsymbol{\eta})+(\hat{\boldsymbol{\gamma}}-\boldsymbol{\gamma}, \boldsymbol{\nabla} v-\boldsymbol{\Pi} \boldsymbol{\eta})+(\boldsymbol{\gamma}, \boldsymbol{\eta}-\Pi \boldsymbol{\eta})
\end{aligned}
$$

for every $\boldsymbol{\eta} \in \mathbf{H}_{h}$ and $v \in W_{h}$. Taking $\boldsymbol{\eta}=\hat{\boldsymbol{\beta}}-\boldsymbol{\beta}_{h} \in \mathbf{H}_{h}$ and $v=\hat{w}-w_{h} \in W_{h}$, we have

$$
\hat{\boldsymbol{\gamma}}-\boldsymbol{\gamma}_{h}=t^{-2}(\boldsymbol{\nabla} v-\boldsymbol{\Pi} \boldsymbol{\eta}),
$$

and inserting this in (3.6), we obtain

$$
\begin{aligned}
a(\hat{\boldsymbol{\beta}}- & \left.\boldsymbol{\beta}_{h}, \hat{\boldsymbol{\beta}}-\boldsymbol{\beta}_{h}\right)+t^{2}\left(\hat{\boldsymbol{\gamma}}-\boldsymbol{\gamma}_{h}, \hat{\boldsymbol{\gamma}}-\boldsymbol{\gamma}_{h}\right) \\
= & a\left(\hat{\boldsymbol{\beta}}-\boldsymbol{\beta}, \hat{\boldsymbol{\beta}}-\boldsymbol{\beta}_{h}\right)+t^{2}\left(\hat{\boldsymbol{\gamma}}-\boldsymbol{\gamma}, \hat{\boldsymbol{\gamma}}-\boldsymbol{\gamma}_{h}\right) \\
& +\left(\boldsymbol{\gamma},\left(\hat{\boldsymbol{\beta}}-\boldsymbol{\beta}_{h}\right)-\boldsymbol{\Pi}\left(\hat{\boldsymbol{\beta}}-\boldsymbol{\beta}_{h}\right)\right) .
\end{aligned}
$$


Therefore, using the coerciveness and continuity of $a($, , , the Schwarz inequality, and the arithmetic geometric mean inequality, we obtain the estimate

$$
\begin{aligned}
& \left\|\hat{\boldsymbol{\beta}}-\boldsymbol{\beta}_{h}\right\|_{1}^{2}+t^{2}\left\|\hat{\boldsymbol{\gamma}}-\boldsymbol{\gamma}_{h}\right\|_{0}^{2} \\
& \quad \leq C\left\{\|\hat{\boldsymbol{\beta}}-\boldsymbol{\beta}\|_{1}^{2}+t^{2}\|\hat{\boldsymbol{\gamma}}-\boldsymbol{\gamma}\|_{0}^{2}+\|\boldsymbol{\gamma}\|_{0}\left\|\left(\hat{\boldsymbol{\beta}}-\boldsymbol{\beta}_{h}\right)-\Pi\left(\hat{\boldsymbol{\beta}}-\boldsymbol{\beta}_{h}\right)\right\|_{0}\right\} .
\end{aligned}
$$

Using (3.2) to bound the last term on the right-hand side, we get (3.5).

From Lemma 3.1 we see that if there exist $\hat{\boldsymbol{\beta}} \in H_{h}$ and $\hat{w} \in W_{h}$ such that $\hat{\boldsymbol{\beta}}$ and $\hat{\boldsymbol{\gamma}}$ are good approximations of $\boldsymbol{\beta}$ and $\boldsymbol{\gamma}$, respectively, we get an error estimate. Therefore, we can state the main result of this section.

Theorem 3.1. Let the spaces $\mathbf{H}_{h} \subset \mathbf{H}_{0}^{1}(\Omega), W_{h} \subset H_{0}^{1}(\Omega), \Gamma_{h} \subset \mathbf{L}^{2}(\Omega)$ and the operator $\Pi$ be such that (3.1) and (3.2) hold. If there exist $\hat{\boldsymbol{\beta}} \in \mathbf{H}_{h}$ and $\hat{w} \in W_{h}$ and an operator $\tilde{\mathbf{\Pi}}: \mathbf{V} \rightarrow \Gamma_{h}$ such that

$$
\begin{gathered}
\|\boldsymbol{\beta}-\hat{\boldsymbol{\beta}}\|_{1} \leq C h\|\boldsymbol{\beta}\|_{2}, \\
\hat{\boldsymbol{\gamma}}=\widetilde{\mathbf{\Pi}} \boldsymbol{\gamma}
\end{gathered}
$$

with $\hat{\boldsymbol{\gamma}}$ defined as in the lemma and

$$
\|\boldsymbol{\eta}-\widetilde{\mathbf{\Pi}} \boldsymbol{\eta}\|_{0} \leq C h\|\boldsymbol{\eta}\|_{1}
$$

for every $\boldsymbol{\eta} \in \mathbf{H}^{1}(\Omega) \cap \mathbf{V}$, then

$$
\left\|\boldsymbol{\beta}-\boldsymbol{\beta}_{h}\right\|_{1}+t\left\|\boldsymbol{\gamma}-\boldsymbol{\gamma}_{h}\right\|_{0} \leq C h\left\{\|\boldsymbol{\beta}\|_{2}+t\|\boldsymbol{\gamma}\|_{1}+\|\boldsymbol{\gamma}\|_{0}\right\} .
$$

Proof. (3.11) follows immediately from Lemma 3.1, (3.8), (3.9), and (3.10).

Corollary 3.1. Under the assumptions of the theorem we have

$$
\left\|w-w_{h}\right\|_{1} \leq C h\left\{\|\boldsymbol{\beta}\|_{2}+t\|\boldsymbol{\gamma}\|_{1}+\|\boldsymbol{\gamma}\|_{0}\right\} .
$$

Proof. We have

$$
\boldsymbol{\nabla} w-\nabla w_{h}=t^{2}\left(\boldsymbol{\gamma}-\boldsymbol{\gamma}_{h}\right)+\boldsymbol{\beta}-\boldsymbol{\Pi} \boldsymbol{\beta}_{h},
$$

hence

$$
\left\|w-w_{h}\right\|_{1} \leq t^{2}\left\|\boldsymbol{\gamma}-\boldsymbol{\gamma}_{h}\right\|_{0}+\|\boldsymbol{\beta}-\boldsymbol{\Pi} \boldsymbol{\beta}\|_{0}+\left\|\boldsymbol{\Pi}\left(\boldsymbol{\beta}-\boldsymbol{\beta}_{h}\right)\right\|_{0} .
$$

Now, from (3.2) we get

$$
\left\|\boldsymbol{\Pi}\left(\boldsymbol{\beta}-\boldsymbol{\beta}_{h}\right)\right\|_{0} \leq C\left\|\boldsymbol{\beta}-\boldsymbol{\beta}_{h}\right\|_{1}
$$

and so, applying again (3.2) and Theorem 3.1, we obtain (3.12).

\section{EXAMPLES}

In this section we show several elements for which our error analysis can be applied.

In the first two examples, condition (3.9) will be satisfied with $\widetilde{\boldsymbol{\Pi}}=\boldsymbol{\Pi}$. In this case, (3.9) can be written in the following way:

$$
\boldsymbol{\Pi}(\boldsymbol{\nabla} w)+\boldsymbol{\Pi}(\hat{\boldsymbol{\beta}}-\boldsymbol{\beta})=\boldsymbol{\nabla} \hat{w} .
$$

Therefore, it is enough to choose the spaces such that there exists a good approximation $\hat{\boldsymbol{\beta}} \in \mathbf{H}_{h}$ of $\boldsymbol{\beta}$ for which a $\hat{w} \in W_{h}$ satisfying (4.1) exists. 
Following the arguments in [7], one can see that (4.1) is satisfied if the spaces and $\Pi$ are chosen in the following way:

$$
\Gamma_{h} \subset \mathbf{H}_{0}(\operatorname{rot}, \Omega)=\left\{\boldsymbol{\mu} \in \mathbf{L}^{2}(\Omega): \operatorname{rot} \boldsymbol{\mu} \in L^{2}(\Omega) \text { and } \boldsymbol{\mu} \cdot \boldsymbol{\tau}=0 \text { on } \partial \Omega\right\},
$$

where

$$
\operatorname{rot} \boldsymbol{\mu}=-\frac{\partial \mu_{2}}{\partial x_{1}}+\frac{\partial \mu_{1}}{\partial x_{2}}
$$

and $\tau$ is the unit tangent to the boundary,

$$
\Pi: \mathbf{H}^{1}(\Omega) \cap \mathbf{H}_{0}(\operatorname{rot}, \Omega) \rightarrow \Gamma_{h} \text { is such that } \int_{\Omega} \operatorname{rot}(\boldsymbol{\eta}-\Pi \boldsymbol{\eta}) q=0
$$

for every $q \in Q_{h}=\operatorname{rot} \Gamma_{h}$,

$$
W_{h} \subset H_{0}^{1}(\Omega) \text { is such that if } \mu \in \Gamma_{h} \text { and } \operatorname{rot} \mu=0 \text {, then }
$$

$$
\boldsymbol{\mu}=\boldsymbol{\nabla} v
$$

for some $v \in W_{h}$, and

$$
\mathbf{H}_{h} \subset \mathbf{H}_{0}^{1}(\Omega) \text { and there exists an interpolation operator }
$$

$$
\mathbf{R}: \mathbf{H}_{0}^{1}(\mathbf{\Omega}) \rightarrow \mathbf{H}_{h}
$$

such that

$$
\int_{\Omega} \operatorname{rot}(\boldsymbol{\beta}-\mathbf{R} \boldsymbol{\beta}) q=0
$$

for every $q \in Q_{h}$ and

$$
\|\boldsymbol{\beta}-\mathbf{R} \boldsymbol{\beta}\|_{1} \leq C h\|\boldsymbol{\beta}\|_{2}
$$

for every $\boldsymbol{\beta} \in \mathbf{H}^{2}(\boldsymbol{\Omega})$.

Indeed, in this case we can take $\hat{\boldsymbol{\beta}}=\mathbf{R} \boldsymbol{\beta}$, and letting $\boldsymbol{\eta}=\boldsymbol{\beta}-\hat{\boldsymbol{\beta}}$ in (4.2) and using (4.4), we get

$$
\int_{\Omega} \operatorname{rot}(\boldsymbol{\beta}-\hat{\boldsymbol{\beta}}) q=\int_{\Omega} \operatorname{rot} \Pi(\boldsymbol{\beta}-\hat{\boldsymbol{\beta}}) q=0
$$

for every $q \in Q_{h}$.

Therefore, taking $q=\operatorname{rot} \Pi(\boldsymbol{\beta}-\hat{\boldsymbol{\beta}})$, we have $\operatorname{rot} \Pi(\boldsymbol{\beta}-\hat{\boldsymbol{\beta}})=0$, which together with (4.3) yields $\Pi(\hat{\boldsymbol{\beta}}-\boldsymbol{\beta})=\nabla v_{1}$ for some $v_{1} \in W_{h}$.

Analogously, we see that $\Pi(\nabla w)=\nabla v_{2}$ for some $v_{2} \in W_{h}$, and so (4.1) is satisfied with $\hat{w}=v_{1}+v_{2}$.

In [7], Bathe, Brezzi, and Fortin obtained error estimates in the limit case $t=0$ under the assumptions (4.2), (4.3), (4.4), and (4.5). Therefore, our analysis extends to the case $t>0$ the results obtained in [5] for the limit problem $t=0$.

We use the standard notation for the spaces of polynomials, that is, $\mathscr{P}_{k}$ is the space of polynomials of degree less than or equal to $k$ and $Q_{i, j}$ is the space of polynomials of degree less than or equal to $i$ in the first variable and to $j$ in the second one. Also, we set $Q_{k}=Q_{k, k}$. 
Example 4.1. A new low-order triangular element. Let $\mathscr{T}_{h}$ be a partition into triangles. We take $\Gamma_{h}$ to be a rotation of the Raviart-Thomas space of the lowest order, namely,

$$
\boldsymbol{\Gamma}_{h}=\left\{\boldsymbol{\mu} \in \mathbf{H}_{0}(\operatorname{rot}, \Omega):\left.\boldsymbol{\mu}\right|_{T} \in \mathscr{P}_{0} \oplus\left(-x_{2}, x_{1}\right) \mathscr{P}_{0}, \forall T \in \mathscr{T}_{h}\right\} .
$$

So, there exists $\Pi$ satisfying (3.2) and (4.2) [12]. We take $W_{h}$ to be the standard space of piecewise linear continuous functions, that is,

$$
W_{h}=\left\{v \in H_{0}^{1}(\Omega):\left.v\right|_{T} \in \mathscr{P}_{1}, \forall T \in \mathscr{T}_{h}\right\},
$$

and so (3.1) and (4.3) can be easily verified.

To define $\mathbf{H}_{h}$, we take a rotation of a space introduced for the Stokes problem [13].

Let $T \in \mathscr{T}_{h}$ and let $\lambda_{1}, \lambda_{2}, \lambda_{3}$ be its barycentric coordinates. We denote by $\tau_{i}$ a unit tangent vector to the side $\lambda_{i}=0$ and define

$$
\mathbf{p}_{1}=\lambda_{2} \lambda_{3} \tau_{1}, \quad \mathbf{p}_{2}=\lambda_{1} \lambda_{3} \tau_{2}, \quad \mathbf{p}_{3}=\lambda_{1} \lambda_{2} \tau_{3}
$$

and

$$
\mathbf{H}_{h}=\left\{\boldsymbol{\eta} \in \mathbf{H}_{0}^{1}(\Omega):\left.\boldsymbol{\eta}\right|_{T} \in \mathscr{P}_{1} \oplus\left\langle\mathbf{p}_{1}, \mathbf{p}_{2}, \mathbf{p}_{3}\right\rangle, \forall T \in \mathscr{T}_{h}\right\},
$$

where $\left\langle\mathbf{p}_{1}, \mathbf{p}_{2}, \mathbf{p}_{3}\right\rangle$ is the space spanned by $\left\{\mathbf{p}_{i}\right\}_{1 \leq i \leq 3}$.

Therefore, the existence of $\mathbf{R}$ satisfying (4.4) follows by a simple rotation and known results [13].

So we can apply Theorem 3.1 and its corollary with $\hat{\boldsymbol{\beta}}=\mathbf{R} \boldsymbol{\beta}$, and we get

$$
\left\|\boldsymbol{\beta}-\boldsymbol{\beta}_{h}\right\|_{1}+t\left\|\boldsymbol{\gamma}-\boldsymbol{\gamma}_{h}\right\|_{0}+\left\|w-w_{h}\right\|_{1} \leq C h\left\{\|\boldsymbol{\beta}\|_{2}+t\|\boldsymbol{\gamma}\|_{1}+\|\boldsymbol{\gamma}\|_{0}\right\} .
$$

When $\Omega$ is a convex polygon it is known (see $[2,9])$ that

$$
\|\boldsymbol{\beta}\|_{2}+t\|\boldsymbol{\gamma}\|_{1}+\|\boldsymbol{\gamma}\|_{0} \leq C\|f\|
$$

and so we obtain an optimal error estimate with constant independent of the plate thickness, namely,

$$
\left\|\boldsymbol{\beta}-\boldsymbol{\beta}_{h}\right\|_{1}+t\left\|\boldsymbol{\gamma}-\boldsymbol{\gamma}_{h}\right\|_{0}+\left\|w-w_{h}\right\|_{1} \leq C h\|f\|_{0} .
$$

Example 4.2. The Bathe-Brezzi elements of second order. In [5], Bathe and Brezzi introduced the following rectangular elements:

$$
\boldsymbol{\Gamma}_{h}=\left\{\boldsymbol{\mu} \in \mathbf{H}_{0}(\operatorname{rot}, \Omega):\left.\boldsymbol{\mu}\right|_{R} \in \widetilde{\mathbf{Q}}, \forall R \in \mathscr{T}_{h}\right\},
$$

where $\widetilde{\mathbf{Q}}=\left\langle 1, x_{1}, x_{2}, x_{1} x_{2}, x_{2}^{2}\right\rangle \times\left\langle 1, x_{1}, x_{2}, x_{1} x_{2}, x_{1}^{2}\right\rangle$ (which is a rotation of the space introduced in [8]),

$$
W_{h}=\left\{v \in H_{0}^{1}(\Omega):\left.v\right|_{R} \in Q_{2}^{r}, \forall R \in \mathscr{T}_{h}\right\},
$$

where $Q_{2}^{r}=\left\langle 1, x_{1}, x_{2}, x_{1} x_{2}, x_{1}^{2}, x_{2}^{2}, x_{1}^{2} x_{2}, x_{1} x_{2}^{2}\right\rangle$, and

$$
\mathbf{H}_{h}=\left\{\boldsymbol{\eta} \in \mathbf{H}_{0}^{1}(\Omega):\left.\boldsymbol{\eta}\right|_{R} \in \mathbf{Q}_{2}, \forall R \in \mathscr{T}_{h}\right\} \text {. }
$$

Clearly, (3.1) and (4.3) hold. The existence of $\boldsymbol{\Pi}$ and $\mathbf{R}$ satisfying (4.2), (4.4), and (4.5) is known [8, 13], and so the error analysis of Lemma 3.1 can be carried out in this case. In order to obtain a second-order estimate, the last term on the right-hand side of (3.7) can be treated as in [5]. So we obtain the 
following error estimate, which generalizes for $t>0$ that obtained in [5] for the case $t=0$ :

$$
\left\|\boldsymbol{\beta}-\boldsymbol{\beta}_{h}\right\|_{1}+t\left\|\boldsymbol{\gamma}-\boldsymbol{\gamma}_{h}\right\|_{0}+\left\|w-w_{h}\right\|_{1} \leq C h^{2}\left\{\|\boldsymbol{\beta}\|_{3}+t\|\boldsymbol{\gamma}\|_{2}+\|\boldsymbol{\gamma}\|_{1}\right\} .
$$

For similar triangular and higher-order elements we refer to [7]. We can apply our analysis to those cases obtaining optimal error estimates.

Example 4.3. The Bathe-Dvorkin elements. Let $\mathscr{T}_{h}$ be a partition into rectangles. Then the Bathe-Dvorkin elements are defined as follows [4, 6]:

$$
\begin{aligned}
& \boldsymbol{\Gamma}_{h}=\left\{\boldsymbol{\mu} \in \mathbf{H}_{0}(\text { rot }, \Omega):\left.\boldsymbol{\mu}\right|_{R} \in Q_{0,1} \times Q_{1,0}, \forall R \in \mathscr{T}_{h}\right\}, \\
& W_{h}=\left\{v \in H_{0}^{1}(\Omega):\left.v\right|_{R} \in Q_{1}, \forall R \in \mathscr{T}_{h}\right\}, \\
& \mathbf{H}_{h}=\left\{\boldsymbol{\eta} \in \mathbf{H}_{0}^{1}(\Omega):\left.\boldsymbol{\eta}\right|_{R} \in \mathbf{Q}_{1}, \forall R \in \mathscr{T}_{h}\right\} .
\end{aligned}
$$

Since $\Gamma_{h}$ is a rotation of the lowest-order Raviart-Thomas space [14], it is known that there exists an interpolation operator $\Pi$ satisfying (4.2) and (3.2) [4, 14]. Also, (3.1) and (4.3) hold, as is easily seen.

In this case we have

$$
Q_{h}=\left\{q \in L^{2}(\Omega):\left.q\right|_{R} \in \mathscr{P}_{0}, \forall R \in \mathscr{T}_{h} \text { and } \int_{\Omega} q=0\right\} .
$$

However, it is known [13] that in this case the operator $\mathbf{R}$ satisfying (4.4) and (4.5) does not exist. Nevertheless, we will prove that (3.9) and (3.10) hold in the case of uniform meshes. More precisely, we assume that the family of meshes $\left\{\mathscr{T}_{h}\right\}$ is obtained by uniform refinement of a starting rectangular mesh in such a way that at each step every element is divided uniformly in sixteen rectangles.

In order to prove (3.9) and (3.10), we need to introduce some notation.

Let $q_{0} \in Q_{h}$ be the checkerboard function, that is, a function which takes the values 1 and -1 alternately in the elements. Let $\widetilde{Q}_{h}$ be the space orthogonal to $q_{0}$, namely,

$$
\widetilde{Q}_{h}=\left\{q \in Q_{h}:\left(q, q_{0}\right)=0\right\},
$$

and let $P: L^{2} \rightarrow \widetilde{Q}_{h}$ be the orthogonal projection.

The following approximation properties for $P$ hold:

$$
\|q-P q\|_{0} \leq C h\|q\|_{1}
$$

for every $q \in H^{1}(\Omega)$ and

$$
\|q-P q\|_{-1} \leq C h\|q\|_{0}
$$

for every $q \in L^{2}(\Omega)$. (Here and thereafter, \|\|$_{-1}$ denotes the norm in the dual space of $H^{1}(\Omega)$.)

Indeed, (4.7) follows from the fact that $\widetilde{Q}_{h}$ contains the piecewise constants over a coarser mesh of size $2 h$, and (4.8) is an easy consequence of (4.7).

Following the arguments in [4], we can prove that for any $\boldsymbol{\beta} \in \mathbf{H}^{s}(\Omega) \cap H_{0}^{1}(\Omega)$, $2 \leq s \leq 3$, there exists $\hat{\boldsymbol{\beta}} \in \mathbf{H}_{h}$ satisfying

$$
\int_{\Omega} \operatorname{rot}(\boldsymbol{\beta}-\hat{\boldsymbol{\beta}}) q=0
$$

for every $q \in \tilde{Q}_{h}$ and

$$
\|\boldsymbol{\beta}-\hat{\boldsymbol{\beta}}\|_{1} \leq C h^{s-2}\|\boldsymbol{\beta}\|_{s} .
$$


Condition (4.9) together with (4.2) yields

$$
\int_{\Omega} \operatorname{rot} \Pi(\boldsymbol{\beta}-\hat{\boldsymbol{\beta}}) q=0
$$

for every $q \in \widetilde{Q}_{h}$.

Now, since

$$
\int_{\Omega} \operatorname{rot} \boldsymbol{\Pi} \hat{\boldsymbol{\beta}} q_{0}=\int_{\Omega} \operatorname{rot} \hat{\boldsymbol{\beta}} q_{0}=0
$$

(see [13]), we have that $\operatorname{rot} \Pi \hat{\boldsymbol{\beta}} \in \widetilde{Q}_{h}$, which together with (4.11) gives

$$
\operatorname{rot} \Pi \hat{\boldsymbol{\beta}}=P \operatorname{rot} \Pi \boldsymbol{\beta} .
$$

For $\boldsymbol{\eta} \in \mathbf{H}_{0}(\operatorname{rot}, \Omega)$ we define $\tilde{\boldsymbol{\Pi}} \boldsymbol{\eta}$ in the following way. Let $\boldsymbol{\chi}(\boldsymbol{\eta}) \in \mathbf{H}_{0}(\operatorname{rot}, \Omega)$ be such that

$$
\operatorname{rot} \chi(\boldsymbol{\eta})=\operatorname{rot} \Pi \eta-P \operatorname{rot} \Pi \eta
$$

and

$$
\|\boldsymbol{\chi}(\boldsymbol{\eta})\|_{s} \leq C\|\operatorname{rot} \Pi \boldsymbol{\eta}-P \operatorname{rot} \Pi \boldsymbol{\eta}\|_{s-1}, \quad s=0,1 .
$$

Take, for example, $\boldsymbol{\chi}(\boldsymbol{\eta})=$ curl $\phi=\left(-\partial \phi / \partial x_{2}, \partial \phi / \partial x_{1}\right)$, where $\phi$ is the solution of the problem

$$
-\Delta \phi=\operatorname{rot} \Pi \eta-P \operatorname{rot} \Pi \eta \quad \text { in } \Omega
$$

with homogeneous Neumann boundary conditions.

Then we set

$$
\widetilde{\boldsymbol{\Pi}} \boldsymbol{\eta}=\boldsymbol{\Pi}(\boldsymbol{\eta}-\boldsymbol{\chi}(\boldsymbol{\eta}))
$$

and show that there exists $\hat{w} \in W_{h}$ such that (3.9) is satisfied with $\hat{\boldsymbol{\beta}}$ defined as above.

Indeed, from $(2.3 b)$ we have

$$
t^{2} \operatorname{rot} \boldsymbol{\gamma}=-\operatorname{rot} \boldsymbol{\beta},
$$

which in view of (4.2) implies

$$
t^{2} \operatorname{rot} \Pi \boldsymbol{\gamma}=-\operatorname{rot} \Pi \beta .
$$

Therefore, using (4.12), we obtain

$$
t^{2} P \operatorname{rot} \Pi \boldsymbol{\gamma}=-\operatorname{rot} \Pi \hat{\boldsymbol{\beta}},
$$

and so, by (4.2) and (4.13) with $\boldsymbol{\eta}=\boldsymbol{\gamma}$, we have

$$
\operatorname{rot} \Pi \chi(\boldsymbol{\gamma})=\operatorname{rot} \Pi \boldsymbol{\gamma}+t^{-2} \operatorname{rot} \Pi \hat{\boldsymbol{\beta}} .
$$

Now the existence of $\hat{w}$, and therefore (3.9), follow from (4.3), (4.15), and (4.16).

Finally, let us verify (3.10). We have

$$
\|\boldsymbol{\eta}-\widetilde{\boldsymbol{\Pi}} \boldsymbol{\eta}\|_{0} \leq\|\boldsymbol{\eta}-\boldsymbol{\Pi} \boldsymbol{\eta}\|_{0}+\|\boldsymbol{\Pi} \boldsymbol{\chi}(\boldsymbol{\eta})\|_{0} .
$$

The first term is bounded by (3.2) while for the second we use (3.2), (4.2), (4.8), and (4.14) to obtain

$$
\begin{aligned}
\|\Pi \chi(\eta)\|_{0} & \leq C h\|\boldsymbol{\chi}(\boldsymbol{\eta})\|_{1}+\|\boldsymbol{\chi}(\boldsymbol{\eta})\|_{0} \\
& \leq C h\|\operatorname{rot} \Pi \boldsymbol{\eta}\|_{0}+C\|\operatorname{rot} \Pi \boldsymbol{\eta}-P \operatorname{rot} \Pi \boldsymbol{\eta}\|_{-1} \\
& \leq C h\|\operatorname{rot} \Pi \boldsymbol{\eta}\|_{0} \leq C h\|\operatorname{rot} \boldsymbol{\eta}\|_{0} \leq C h\|\boldsymbol{\eta}\|_{1} .
\end{aligned}
$$


Therefore, we can apply Theorem 3.1 and its corollary, with (3.8) replaced by (4.10) with $s=3$, to obtain the error estimate

$$
\left\|\boldsymbol{\beta}-\boldsymbol{\beta}_{h}\right\|_{1}+t\left\|\boldsymbol{\gamma}-\boldsymbol{\gamma}_{h}\right\|_{0}+\left\|w-w_{h}\right\|_{1} \leq C h\left\{\|\boldsymbol{\beta}\|_{3}+t\|\boldsymbol{\gamma}\|_{1}+\|\boldsymbol{\gamma}\|_{0}\right\} .
$$

Remark 4.1. The estimate (4.17) improves the one obtained in [4], which required $\boldsymbol{\gamma} \in \mathbf{H}^{2}(\Omega)$.

Taking $s=\frac{5}{2}$ in (4.10), we also obtain

$$
\left\|\boldsymbol{\beta}-\boldsymbol{\beta}_{h}\right\|_{1}+t\left\|\boldsymbol{\gamma}-\boldsymbol{\gamma}_{h}\right\|_{0}+\left\|w-w_{h}\right\|_{1} \leq C h^{1 / 2}\left\{\|\boldsymbol{\beta}\|_{5 / 2}+t\|\boldsymbol{\gamma}\|_{1}+\|\boldsymbol{\gamma}\|_{0}\right\} .
$$

When $\Omega$ has a smooth boundary, it is known (see [3]) that the norms on the right-hand side of (4.18) are bounded uniformly in $t$. The natural extension of the results in [2] to a square domain, together with (4.18), would provide an $O\left(h^{1 / 2}\right)$ error estimate uniform in the plate thickness.

It would be very interesting to relax also the regularity requirement on $\boldsymbol{\beta}$ in order to obtain optimal-order convergence independently of the plate thickness.

\section{The NONCONFORMing ELEMENTS OF ARNOLd AND FALK}

In this section we extend the error analysis of $\S 3$ to the Arnold and Falk method [2], in which the transverse displacement $w$ is approximated by nonconforming elements.

The Arnold and Falk elements are defined as follows. Let $\mathscr{T}_{h}$ be a partition of $\Omega$ into triangles; then

$$
\begin{array}{r}
\boldsymbol{\Gamma}_{h}=\left\{\boldsymbol{\mu} \in \mathbf{L}^{2}(\mathbf{\Omega}):\left.\boldsymbol{\mu}\right|_{T} \in \mathscr{P}_{0}, \forall T \in \mathscr{T}_{h}\right\}, \\
W_{h}=\left\{v \in L^{2}(\Omega):\left.v\right|_{T} \in \mathscr{P}_{1}, \forall T \in \mathscr{T}_{h}, \text { and } v\right. \text { is continuous at } \\
\text { midpoints of element edges and vanishes at } \\
\text { midpoints of boundary edges }\},
\end{array}
$$

and

$$
\mathbf{H}_{h}=\left\{\boldsymbol{\eta} \in \mathbf{H}_{0}^{1}(\Omega):\left.\boldsymbol{\eta}\right|_{\mathbf{T}} \in \mathscr{P}_{1} \oplus \mathscr{P}_{0} b_{T}, \forall T \in \mathscr{T}_{h}\right\},
$$

where $b_{T}$ is a bubble function of degree 3 , namely, $b_{T} \in \mathscr{P}_{3}$ and $b_{T}=0$ on $\partial T$.

For $v \in W_{h}$, let $\nabla_{h} v \in \mathbf{L}^{2}(\Omega)$ be the piecewise constant vector function whose restriction to each $T \in \mathscr{T}_{h}$ is given by $\left.\nabla v\right|_{T}$.

Let $\mathbf{P}: \mathbf{L}^{2}(\Omega) \rightarrow \Gamma_{h}$ be the $\mathbf{L}^{2}$-projection. Then the approximation of [2] is obtained by replacing $\boldsymbol{\nabla}$ by $\nabla_{h}$ and taking $\Pi=\mathbf{P}$ in (3.3). That is, $\left(\boldsymbol{\beta}_{h}, w_{h}, \boldsymbol{\gamma}_{h}\right) \in \mathbf{H}_{h} \times W_{h} \times \Gamma_{h}$ satisfy

$$
a\left(\boldsymbol{\beta}_{h}, \boldsymbol{\eta}\right)+\left(\boldsymbol{\gamma}_{h}, \nabla_{h} v-\mathbf{P} \boldsymbol{\eta}\right)=(f, v)
$$

and

$$
\boldsymbol{\gamma}_{h}=t^{-2}\left(\nabla_{h} w_{h}-\mathbf{P} \boldsymbol{\beta}_{h}\right)
$$

for every $\boldsymbol{\eta} \in \mathbf{H}_{h}$ and $v \in W_{h}$.

Since $W_{h} \not \subset H_{0}^{1}(\Omega)$, the error equation includes consistency terms, and therefore (3.4) is modified as follows:

$$
a\left(\boldsymbol{\beta}-\boldsymbol{\beta}_{h}, \boldsymbol{\eta}\right)+\left(\boldsymbol{\gamma}-\boldsymbol{\gamma}_{h}, \nabla_{h} v-\mathbf{P} \boldsymbol{\eta}\right)=(\boldsymbol{\gamma}, \boldsymbol{\eta}-\mathbf{P} \boldsymbol{\eta})+\sum_{T \in \mathscr{T}_{h}} \int_{\partial T} v \boldsymbol{\gamma} \cdot \mathbf{n}_{T}
$$


for every $\boldsymbol{\eta} \in \mathbf{H}_{h}$ and $v \in W_{h}$, where $\mathbf{n}_{T}$ is the outer unit normal to the boundary of $T$.

Proceeding as in Lemma 3.1, we obtain

$$
\begin{aligned}
\left\|\hat{\boldsymbol{\beta}}-\boldsymbol{\beta}_{h}\right\|_{1}^{2}+t^{2}\left\|\hat{\boldsymbol{\gamma}}-\boldsymbol{\gamma}_{h}\right\|_{0}^{2} \leq & C\left\{\left\|\hat{\boldsymbol{\beta}}-\boldsymbol{\beta}_{h}\right\|_{1}^{2}+t^{2}\|\hat{\boldsymbol{\gamma}}-\boldsymbol{\gamma}\|_{0}^{2}+h^{2}\|\boldsymbol{\gamma}\|_{0}^{2}\right\} \\
& +\left|\sum_{T \in \mathscr{S}_{h}} \int_{\partial T} v \boldsymbol{\gamma} \cdot \mathbf{n}_{T}\right|
\end{aligned}
$$

for $\hat{\boldsymbol{\beta}} \in \mathbf{H}_{h}, \hat{w} \in W_{h}, \hat{\boldsymbol{\gamma}}=t^{-2}\left(\nabla_{h} \hat{w}-\mathbf{P} \hat{\boldsymbol{\beta}}\right) \in \boldsymbol{\Gamma}_{h}$, and $v=\hat{w}-w_{h} \in W_{h}$.

In order to estimate the last term on the right-hand side of (5.2), we use the following lemma due to Crouzeix and Raviart [12], which is crucial for the analysis on nonconforming methods.

Lemma 5.1. Let $\phi \in \mathbf{H}^{1}(\Omega)$ and $v \in W_{h}$; then

$$
\left|\sum_{T \in \mathscr{T}_{h}} \int_{\partial T} v \boldsymbol{\phi} \cdot \mathbf{n}_{T}\right| \leq C h\|\phi\|_{1}\left\|\nabla_{h} v\right\|_{0} .
$$

However, a direct application of this lemma would give an estimate which depends on $\|\boldsymbol{\gamma}\|_{1}$, which is not bounded uniformly in $t$ [3]. Therefore, we have to proceed in a different way to obtain a modification of Lemma 3.1.

Since $\Omega$ is simply connected, $\gamma$ can be written as

$$
\boldsymbol{\gamma}=\boldsymbol{\nabla} \boldsymbol{r}+\operatorname{curl} p,
$$

where $r \in H_{0}^{1}(\Omega)$ and $p \in H^{1}(\Omega)$ with $\int_{\Omega} p=0$.

Lemma 5.2. Let $\hat{\boldsymbol{\beta}} \in \mathbf{H}_{h}, \hat{w} \in W_{h}$, and $\hat{\boldsymbol{\gamma}}=t^{-2}\left(\nabla_{h} \hat{w}-\mathbf{P} \hat{\boldsymbol{\beta}}\right) \in \boldsymbol{\Gamma}_{h}$; then

$$
\begin{aligned}
\left\|\hat{\boldsymbol{\beta}}-\boldsymbol{\beta}_{h}\right\|_{1}+t\left\|\hat{\boldsymbol{\gamma}}-\boldsymbol{\gamma}_{h}\right\|_{0} \leq C\left\{\|\hat{\boldsymbol{\beta}}-\boldsymbol{\beta}\|_{1}+t\|\hat{\boldsymbol{\gamma}}-\boldsymbol{\gamma}\|_{0}\right. \\
\left.+h\left(\|r\|_{2}+\|p\|_{1}+t\|p\|_{2}\right)\right\} .
\end{aligned}
$$

Proof. Applying Lemma 5.1, we get

$$
\left|\sum_{T \in \mathscr{T}_{h}} \int_{\partial T} v \nabla r \cdot \mathbf{n}_{T}\right| \leq C h\|r\|_{2}\left\|\nabla_{h} v\right\|_{0},
$$

and since

$$
\boldsymbol{\nabla}_{h} v=t^{2}\left(\hat{\boldsymbol{\gamma}}-\boldsymbol{\gamma}_{h}\right)+\mathbf{P}\left(\hat{\boldsymbol{\beta}}-\boldsymbol{\beta}_{h}\right)
$$

we obtain

$$
\left|\sum_{T \in \mathscr{T}_{h}} \int_{\partial T} v \nabla r \cdot \mathbf{n}_{T}\right| \leq C h\|r\|_{2}\left(t^{2}\left\|\hat{\boldsymbol{\gamma}}-\boldsymbol{\gamma}_{h}\right\|_{0}+\left\|\hat{\boldsymbol{\beta}}-\boldsymbol{\beta}_{h}\right\|_{0}\right) .
$$

On the other hand, we have

$$
\sum_{T \in \mathscr{T}_{h}} \int_{\partial T} v \operatorname{curl} p \cdot \mathbf{n}_{T}=\sum_{T \in \mathscr{T}_{h}} \int_{T} \operatorname{curl} p \cdot \nabla_{h} v .
$$

Let $\hat{p} \in H^{1}(\Omega)$ be a continuous piecewise linear approximation of $p$ such that

$$
\|p-\hat{p}\|_{1} \leq C h\|p\|_{2},
$$




$$
\|p-\hat{p}\|_{0} \leq C h\|p\|_{1}
$$

and

$$
\|\hat{p}\|_{1} \leq C\|p\|_{1}
$$

(for example, take $\hat{p}$ to be the regularized interpolation of Clement ; see [13]).

It is easily seen that

$$
\sum_{T \in \mathscr{T}_{h}} \int_{T} \operatorname{curl} \hat{p} \cdot \nabla_{h} v=0
$$

and so we get from (5.4) and (5.6) that

$$
\sum_{T \in \mathscr{T}_{h}} \int_{\partial T} v \operatorname{curl} p \cdot \mathbf{n}_{T}=\left(\operatorname{curl}(p-\hat{p}), t^{2}\left(\hat{\boldsymbol{\gamma}}-\boldsymbol{\gamma}_{h}\right)+\mathbf{P}\left(\hat{\boldsymbol{\beta}}-\boldsymbol{\beta}_{h}\right)\right) .
$$

Now using (5.7), we have

$$
\left|\left(\operatorname{curl}(p-\hat{p}), t^{2}\left(\boldsymbol{\gamma}-\boldsymbol{\gamma}_{h}\right)\right)\right| \leq C h\|p\|_{2} T^{2}\left\|\hat{\boldsymbol{\gamma}}-\boldsymbol{\gamma}_{h}\right\|_{0},
$$

while to estimate the other term in (5.10), we decompose it as follows:

$$
\left(\operatorname{curl}(p-\hat{p}), \mathbf{P}\left(\hat{\boldsymbol{\beta}}-\boldsymbol{\beta}_{h}\right)-\left(\hat{\boldsymbol{\beta}}-\boldsymbol{\beta}_{h}\right)\right)+\left(\operatorname{curl}(p-\hat{p}), \hat{\boldsymbol{\beta}}-\boldsymbol{\beta}_{h}\right) .
$$

Now, using (5.9) and known approximation properties for the $L^{2}$-projection, we get

$$
\left|\left(\operatorname{curl}(p-\hat{p}), \mathbf{P}\left(\hat{\boldsymbol{\beta}}-\boldsymbol{\beta}_{h}\right)-\left(\hat{\boldsymbol{\beta}}-\boldsymbol{\beta}_{h}\right)\right)\right| \leq C\|p\|_{1} h\left\|\hat{\boldsymbol{\beta}}-\boldsymbol{\beta}_{h}\right\|_{1},
$$

and using (5.8), we obtain

$$
\begin{aligned}
\mid\left(\operatorname{curl}(p-\hat{p}), \hat{\boldsymbol{\beta}}-\boldsymbol{\beta}_{h}\right) & =\left|\left(p-\hat{p}, \operatorname{rot}\left(\hat{\boldsymbol{\beta}}-\boldsymbol{\beta}_{h}\right)\right)\right| \\
& \leq C h\|p\|_{1}\left\|\hat{\boldsymbol{\beta}}-\boldsymbol{\beta}_{h}\right\|_{1} .
\end{aligned}
$$

Therefore, collecting all the estimates, we obtain

$$
\left|\sum_{T \in \mathscr{T}_{h}} \int_{\partial T} v \operatorname{curl} p \cdot \mathbf{n}_{T}\right| \leq C h\left\{\|p\|_{2} t^{2}\left\|\hat{\boldsymbol{\gamma}}-\boldsymbol{\gamma}_{h}\right\|_{0}+\|p\|_{1}\left\|\hat{\boldsymbol{\beta}}-\boldsymbol{\beta}_{h}\right\|_{1}\right\} .
$$

Now, from (5.5) and (5.11) we get

$$
\left|\sum_{T \in \mathscr{T}_{h}} \int_{\partial T} v \boldsymbol{\gamma} \cdot \mathbf{n}_{T}\right| \leq C h\left(\|r\|_{2}+t\|p\|_{2}+\|p\|_{1}\right)\left(t\left\|\hat{\boldsymbol{\gamma}}-\boldsymbol{\gamma}_{h}\right\|_{0}+\left\|\hat{\boldsymbol{\beta}}-\boldsymbol{\beta}_{h}\right\|_{1}\right),
$$

which together with (5.2) yields (5.3), and so the lemma is proved.

Now, as in the conforming case, it is enough to see that there exists $\hat{\boldsymbol{\beta}} \in \mathbf{H}_{h}$ satisfying

$$
\|\boldsymbol{\beta}-\hat{\boldsymbol{\beta}}\|_{1} \leq C h\|\boldsymbol{\beta}\|_{2}
$$

and $\hat{w} \in W_{h}$ such that $\hat{\boldsymbol{\gamma}}=\mathbf{P} \boldsymbol{\gamma}$, that is,

$$
\mathbf{P}(\boldsymbol{\nabla} w)+\mathbf{P}(\hat{\boldsymbol{\beta}}-\boldsymbol{\beta})=\nabla_{h} \hat{w} .
$$

First, we take $\hat{\boldsymbol{\beta}}=\mathbf{R} \boldsymbol{\beta}$, where $\mathbf{R}$ is the interpolation defined in [1], which satisfies

$$
\int_{T}(\boldsymbol{\beta}-\mathbf{R} \boldsymbol{\beta}) \cdot \mathbf{q}=0
$$


for every $\mathbf{q} \in \mathscr{P}_{0}$ and every $T \in \mathscr{T}_{h}$. Therefore, (5.12) is satisfied [1] and $\mathbf{P}(\hat{\boldsymbol{\beta}}-\boldsymbol{\beta})=\mathbf{0}$. So, (5.13) will hold if there exists $\hat{w} \in W_{h}$ such that $\mathbf{P}(\nabla w)=$ $\nabla_{h} \hat{w}$, or

$$
\int_{T}\left(\nabla w-\nabla_{h} \hat{w}\right) \cdot \mathbf{q}=0
$$

for every $\mathbf{q} \in \mathscr{P}_{0}$ and every $T \in \mathscr{T}_{h}$. But (5.14) is equivalent to

$$
\int_{\partial T}(w-\hat{w}) \mathbf{q} \cdot \mathbf{n}_{T}=0 .
$$

Now, let $l$ be a side of $T$ and let $M_{l}$ be the midpoint of $l$. We take $\hat{w} \in W_{h}$ such that

$$
\hat{w}\left(M_{l}\right)=\frac{1}{|l|} \int_{l} w,
$$

where $|l|$ is the length of $l$, and therefore (5.15), and consequently (5.13), are clearly satisfied.

Then, applying Lemma 5.2, we obtain the error estimate

$$
\left\|\boldsymbol{\beta}-\boldsymbol{\beta}_{h}\right\|_{1}+t\left\|\boldsymbol{\gamma}-\boldsymbol{\gamma}_{h}\right\|_{0} \leq C h\left\{\|\boldsymbol{\beta}\|_{2}+\|r\|_{2}+\|p\|_{1}+t\|p\|_{2}\right\}
$$

and consequently,

$$
\left\|\nabla w-\nabla_{h} w_{h}\right\|_{0} \leq C h\left\{\|\boldsymbol{\beta}\|_{2}+\|r\|_{2}+\|p\|_{1}+t\|p\|_{2}\right\} .
$$

If $\Omega$ is a convex polygon, the right-hand side of (5.16) is bounded by $C\|f\|_{0}$ $[2,9]$, and therefore

$$
\left\|\boldsymbol{\beta}-\boldsymbol{\beta}_{h}\right\|_{1}+t\left\|\boldsymbol{\gamma}-\boldsymbol{\gamma}_{h}\right\|_{0}+\left\|\nabla w-\nabla_{h} w\right\|_{0} \leq C h\|f\|_{0} .
$$

\section{ACKNOWLEDGMENTS}

We want to thank M. A. Muschietti and the referee for helpful comments.

Part of this work was done while the first author was visiting the Center for Applied Mathematics at Purdue University. He wishes to express his gratitude to this institution for its hospitality.

\section{BIBLIOGRAPHY}

1. D. N. Arnold, F. Brezzi, and M. Fortin, A stable finite element for the Stokes equations, Calcolo 21 (1984), 337-344.

2. D. N. Arnold and R. S. Falk, A uniformly accurate finite element method for the ReissnerMindlin plate, SIAM J. Numer. Anal. 26 (1989), 1276-1290.

3. _ The boundary layer for the Reissner-Mindlin plate model, SIAM J. Math. Anal. 21 (1990), 281-312.

4. K. J. Bathe and F. Brezzi, On the convergence of a four-node plate bending element based on Mindlin-Reissner plate theory and a mixed interpolation, MAFELAP V (J. R. Whiteman, ed.), London, 1985, pp. 491-503.

5. __ A simplified analysis of two plate bending elements - the MITC4 and MITC 9 elements, NUMETA 87 (G. N. Pande and J. Middleton, eds.), Numerical Techniques for Engineering Analysis and Design, vol. 1, Martinus Nijhoff, Dordrecht, 1987.

6. K. J. Bathe and E. N. Dvorkin, A four-node plate bending element based on Mindlin-Reissner plate theory and a mixed interpolation, J. Numer. Methods Engrg. 21 (1985), 367-383.

7. F. Brezzi, K. J. Bathe, and M. Fortin, Mixed-interpolated elements for Reissner-Mindlin plates, Internat. J. Numer. Methods Engrg. 28 (1989), 1787-1801. 
8. F. Brezzi, J. Douglas, Jr., M. Fortin, and L. D. Marini, Efficient rectangular mixed finite elements in two and three space variables, Model. Math. Anal. Numér. 21 (1987), 581-604.

9. F. Brezzi and M. Fortin, Numerical approximation of Mindlin-Reissner plates, Math. Comp. 47 (1986), 151-158.

10. __ Hybrid and mixed finite element methods (to appear).

11. P. Ciarlet, The finite element method for elliptic problems, North-Holland, Amsterdam, 1978.

12. M. Crouzeix and P. A. Raviart, Conforming and nonconforming finite element methods for solving the stationary Stokes equations, RAIRO Anal. Numér. R-3 (1973), 33-76.

13. V. Girault and P. A. Raviart, Finite element methods for Navier-Stokes equations, Lecture Notes in Math., vol. 749, Springer, 1986.

14. P. A. Raviart and J. M. Thomas, A mixed finite element method for second order elliptic problems, Mathematical Aspects of the Finite Element Method, Lecture Notes in Math., vol. 606, Springer, 1977, pp. 292-315.

Departamento de Matemática, Facultad de Ciencias Exactas, Universidad Nacional de la Plata, Casilla de Correo 172, 1900 la Plata, Argentina 